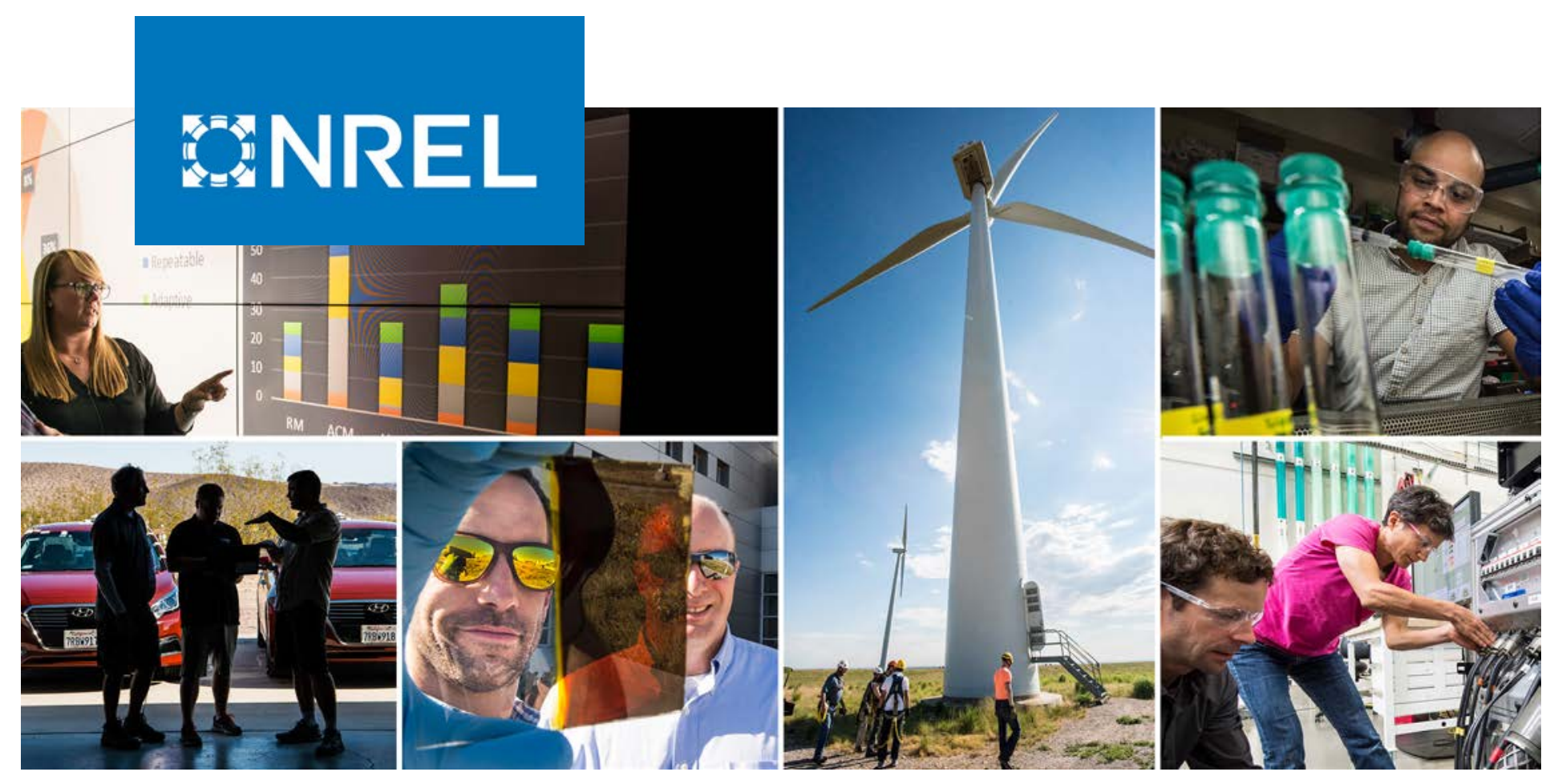

\title{
Post-Disturbance Dynamic Distribution System Restoration with DGs and Mobile Resources
}

\section{Preprint}

Weijia Liu, Fei Ding, Utkarsh Kumar, and Shuva Paul

National Renewable Energy Laboratory

Presented at the 2020 IEEE Power and Energy Society General Meeting (IEEE PES GM)

August 3-6, 2020

NREL is a national laboratory of the U.S. Department of Energy

Office of Energy Efficiency \& Renewable Energy

Operated by the Alliance for Sustainable Energy, LLC

This report is available at no cost from the National Renewable Energy Laboratory (NREL) at www.nrel.gov/publications.

\section{Conference Paper}

NREL/CP-5D00-75372

August 2020 


\title{
GHREL
}

\section{Post-Disturbance Dynamic Distribution System Restoration with DGs and Mobile Resources}

\section{Preprint}

\author{
Weijia Liu, Fei Ding, Utkarsh Kumar, and Shuva Paul \\ National Renewable Energy Laboratory
}

\author{
Suggested Citation \\ Liu, Weijia, Fei Ding, Utkarsh Kumar, and Shuva Paul. 2020. Post-Disturbance Dynamic \\ Distribution System Restoration with DGs and Mobile Resources: Preprint. Golden, CO: \\ National Renewable Energy Laboratory. NREL/CP-5D00-75372. \\ https://www.nrel.gov/docs/fy20osti/75372.pdf.
}

\begin{abstract}
(C) 2020 IEEE. Personal use of this material is permitted. Permission from IEEE must be obtained for all other uses, in any current or future media, including reprinting/republishing this material for advertising or promotional purposes, creating new collective works, for resale or redistribution to servers or lists, or reuse of any copyrighted component of this work in other works.
\end{abstract}

NREL is a national laboratory of the U.S. Department of Energy Office of Energy Efficiency \& Renewable Energy Operated by the Alliance for Sustainable Energy, LLC

This report is available at no cost from the National Renewable Energy Laboratory (NREL) at www.nrel.gov/publications.

Contract No. DE-AC36-08GO28308
Conference Paper

NREL/CP-5D00-75372

August 2020

National Renewable Energy Laboratory 15013 Denver West Parkway Golden, CO 80401

303-275-3000 • www.nrel.gov 


\section{NOTICE}

This work was authored in part by the National Renewable Energy Laboratory, operated by Alliance for Sustainable Energy, LLC, for the U.S. Department of Energy (DOE) under Contract No. DE-AC36-08GO28308. Funding provided by NREL's Laboratory Directed Research and Development Program. The views expressed herein do not necessarily represent the views of the DOE or the U.S. Government.

This report is available at no cost from the National Renewable Energy Laboratory (NREL) at www.nrel.gov/publications.

U.S. Department of Energy (DOE) reports produced after 1991 and a growing number of pre-1991 documents are available free via www.OSTI.gov.

Cover Photos by Dennis Schroeder: (clockwise, left to right) NREL 51934, NREL 45897, NREL 42160, NREL 45891, NREL 48097, NREL 46526.

NREL prints on paper that contains recycled content. 


\title{
Post-Disturbance Dynamic Distribution System Restoration with DGs and Mobile Resources
}

\author{
Weijia Liu, Fei Ding, Utkarsh Kumar, Shuva Paul \\ National Renewable Energy Laboratory \\ Golden, CO, U.S.A. \\ \{weijia.liu; fei.ding; utkarsh.kumar; shuva.paul\}@nrel.gov
}

\begin{abstract}
Distributed generations (DGs) can act as emergency power supplies when distribution systems suffer from outages. However, the generation capabilities of DGs are generally limited by a number of factors including weather conditions, fuel limitations, etc. In this context, mobile resources that are able to reallocate resources to desired locations are regarded as important complements to conventional fixed DGs in assisting distribution system restoration. In this paper, a distribution system restoration model with DGs and mobile resources is proposed. Firstly, the dispatch and allocation of mobile resources are modeled with respect to the characteristics of the traffic network. Then the developed mobile resource models are integrated into the distribution system restoration model to co-optimize the scheduling of DGs and mobile resources. Uncertainty factors are managed by a model predictive control approach so that system operators can dynamically adjust the restoration strategy with the up-to-date information. The effectiveness of the proposed method is validated through an IEEE 13-bus test system.
\end{abstract}

Index Terms--Distribution system restoration, distributed generations (DGs), mobile resources, model predictive control (MPC)

\section{INTRODUCTION}

The recovery from power outages has always been an important topic in power systems around the world. With the growing need of establishing more reliable and resilient power grids in recent years, reasonable power system restoration strategies are in urgent demand to reduce social and economic damages caused by blackouts [1].

Power system outages can be categorized into two kinds, transmission system outages and distribution system outages. Transmission system restoration utilizes conventional blackstart generators to rebuild the backbone network and have been extensively investigated. However, distribution system restoration does not witness significant progress due to the lack of power generation resources before the vast deployment of distributed generations (DGs) becomes a reality. The integration of DGs enables the outage distribution system to provide a limited amount of power generation capacity to sustain power supply. For example, the contributions of distributed energy storage units were studied in an agent-based framework in [2]. The cold load pickup characteristics are integrated into a DG-based restoration strategy in [3]. The concept of microgrid were employed to formulate distribution restoration strategies in [4]. A decentralized distribution system restoration framework is introduced in [5]. The optimal dispatch and control of DGs during distribution system restoration is studied in [6]. In these research works, distribution system operators will formulate restoration strategies with respect to the available generation capacities of DGs.

Meanwhile, the generation capacities of DGs are generally limited in space and time. In this context, mobile resources that are not installed at a fixed location have attracted research attention. Mobile resources, such as mobile generators and mobile batteries, can provide emergency power supply after an outage. As a complement to fixed DGs, mobile resources can be allocated to locations that have no or limited access to DG power. So far, the consideration of mobile resources is limited to the pre-positioning or one-time reallocation of mobile generating resources at optimized nodes to deal with potential natural disasters [7]-[9]. In these works, the locations of mobile resources will be optimized and remain unchanged for the entire restoration horizon. However, the allocation of mobile resources is deeply influenced by the conditions of the traffic network and the status of distribution system outages, where both factors are time-variant and difficult to predict.

In this context, this paper integrates the traffic network constraint and the mobile resources into distribution system restoration problems in the post-disturbance scenario to fully utilize the potentials of mobile resources. The mobile resources are modeled based on traveling behaviors and traveling time within the corresponding traffic network. The uncertainty factors such as traffic conditions, renewable energy forecasts, and fault information in the distribution systems are managed by a model predictive control (MPC) approach. With the developed MPCbased optimization algorithm, distribution system operators can dynamically modify and update system restoration strategy and mobile resource dispatch with up-to-date information.

The rest of this paper is organized as follows. Section II models the transportation of mobile resources. Section III integrates mobile resources into the MPC-based distribution system restoration model. Section IV demonstrates the results based on the proposed method. Section V concludes the paper.

\section{MobiLE RESOURCE TRANSPORTATION MODEL}

\section{A. Transportation Network Modeling}

The existence of mobile resources enables system operators to reallocate generating resources to reduce the negative 
impacts of distribution system outages. Meanwhile, the dispatch of mobile resources is not only constrained by their corresponding characteristics but also limited by the status of the transportation network.

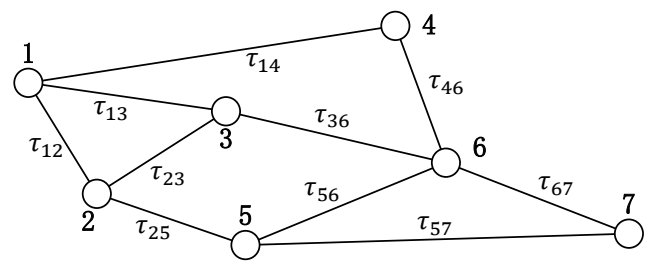

Fig. 1. Demonstration of a simple weighted traffic network.

Take the demonstration of a simple traffic network in Fig. 1 as an example. There are multiple routes available to move resources from one traffic node to another, and it is reasonable to assume that the fleet will use the available path with the shortest distance or the least traveling time. In Fig. 1, the transportation time is selected as the weights for the corresponding traffic network and the path with the least traveling time can be easily obtained by the widely used Dijkstra algorithm [10]. For simplicity, the matrix of least traveling time for a traffic network with $N$ nodes, denoted by $\mathbf{T}$, is presented in (1) where $\tau_{i j}$ denotes the least traveling time between nodes $i$ and $j$. Note that $\mathbf{T}$ is not constant and subject to changes if certain paths become unavailable (e.g., congestion or bad weather).

$$
\mathbf{T}=\left[\tau_{i j}\right]_{N \times N}
$$

The traffic network limitation $\mathbf{T}$ should be integrated into the dispatch of mobile resources to regulate the temporal behaviors of both the mobile resources and the outage systems. Note that the traveling time $\tau_{i j}$ is a continuous parameter, while power system restoration models usually employs discretized time steps such as $15 \mathrm{~min}$ and 1 hour. Assume the time interval of power system restoration models is $\Delta t$, the least transportation time $\tau_{i j}$ in (1) should be discretized by (2):

$$
\Delta \tau_{i j}=\left\lceil\tau_{i j} / \Delta t\right\rceil^{+}
$$

where $\Delta \tau_{i j}$ is the discretized time steps and $\left\lceil\mathrm{T}^{+}\right.$denotes the ceiling function, respectively.

\section{B. Mobile Resource Transportation Model}

When suffering from power outages, mobile resources such as diesel fuel transportation fleet (DFTF), mobile energy storage fleet (MESF), mobile emergency generating units, and electric vehicles with vehicle-to-grid capabilities may be available to distribution system operators. In this paper, DFTF and MESF are investigated in detail for the following reasons:

1) Diesel generators are important backup resources in distribution systems. However, the fuel reserve of diesel generators may be limited when blackout occurs. Hence, diesel generators cannot contribute to the power supply recovery unless the diesel fuel is abundant or reasonably reallocated, which requires the proper dispatch of DFTF.

2) In addition to diesel generators, emergency response vehicles such as MESF are available to utilities companies in Europe and North America [8], [11]-[12]. Moreover, the MESF model also applies to other emergency power suppliers such as electric vehicles.
Although DFTF, MESF, and other mobile resources may have different operating characteristics, they utilize the same traffic network and thus following the identical constraints from the transportation perspective. With discretized transportation time $\Delta \tau_{i j}$, the transportation of mobile resources can be modeled by the following constraints employing a similar linearization technique for unit commitment [13].

$$
\begin{gathered}
y_{i, t}=y_{i, t-1}+\sum_{j \in \Phi_{\mathrm{N}, \mathrm{C}}} y_{j i, t}^{\mathrm{A}}-\sum_{j \in \Phi_{\mathrm{N}, \mathrm{C}}} y_{i j, t}^{\mathrm{D}} \\
\sum_{\tau=t}^{t+\Delta \tau} y_{i, t} \geq \Delta \tau\left(y_{i, t}-y_{i, t-1}\right) \\
\sum_{i \in \Phi_{\mathrm{N}, \mathrm{C}}} y_{i, t} \leq 1 \\
y_{i j, t}^{\mathrm{D}} \geq y_{i, t-1}-y_{i, t} \\
y_{i j, t}^{\mathrm{D}} \leq y_{i, t-1} \\
y_{i j, t+\Delta \tau_{i j}}^{\mathrm{A}}=y_{i j, t}^{\mathrm{D}} \\
0 \leq y_{i, t} \leq 1 \\
y_{i j, t}^{\mathrm{A}}, y_{i j, t}^{\mathrm{D}} \in\{0,1\}
\end{gathered}
$$

where $y_{i, t}$ is a continuous variable denoting the availability status of a given mobile resource fleet. If the mobile fleet parked at node $i$ at time $t, y_{i, t}=1 . y_{i j, t}^{\mathrm{A}}$ and $y_{i j, t}^{\mathrm{D}}$ are Boolean variables denoting the arrival/departure of the mobile fleet from node $i$ to node $j$ at time $t$, respectively. $\Phi_{\mathrm{N}, \mathrm{C}}$ represents the set of candidate traffic nodes where the mobile fleet can travel to. $\Delta \tau$ denotes the discretized loading/unloading time.

In the above transportation model, equation (3) calculates the parking status of the mobile fleet. Constraint (4) enforces that each mobile fleet must remain parked for at least $\Delta \tau$ time intervals to finish loading/unloading before setting off to a new destination. Constraint (5) enforces that the mobile fleet cannot be parked at more than one traffic nodes simultaneously. Constraints (6) and (7) guarantee that the departure of a mobile fleet from node $i$ is feasible only if the same fleet is already parked at node $i$. Equation (8) denotes that the departed mobile fleet will arrive at the destination nodes after the discretized time interval $\Delta \tau_{i j}$. Constraint (9) enforces that $y_{i, t}$ ranges from 0 to 1 . As a result, although $y_{i, t}$ is defined as a continuous variable, it will only take Boolean values based on constraints (3)-(10).

In addition to constraints (3)-(10) that apply to all mobile resources, the transportation of MESF is different from DFTF in the following two aspects: 1) the MESF can be dispatched to all traffic nodes while the transportation of DFTF is limited among locations with diesel generators; 2) multiple DFTF vehicles can be parked at the same node, while each node can accommodate at most one MESF simultaneously. Constraint (11) is introduced to MESF transportation models to guarantee that each node cannot have more than one MESF at the same time.

$$
\sum_{w \in \Phi_{\mathrm{ME}}} y_{w, i, t} \leq 1, \forall i \in \Phi_{\mathrm{N}}
$$

where $\Phi_{\mathrm{ME}}$ denotes the set of MESFs and $\Phi_{\mathrm{N}}$ denotes the set of all nodes in the distribution system. In this paper, subscripts $v$ and $w$ respectively denote the index of DFTF and MESF, unless otherwise specified.

Note that this section only models the transportation of DFTFs and MESFs. Other constraints such as charging/discharging of MESFs and diesel capacity limitations of DFTFs will be modeled in Section III. 


\section{INTEGRATED RESTORATION MODEL}

\section{A. Integrated Restoration Framework}

After an outage occurs in a distribution system, system operators are responsible for restoring power supply to end customers utilizing available resources. In traditional cases where mobile resources are not integrated, operators only solve a restoration model with one module, i.e., a distribution system module. On the other hand, the consideration of mobile resource dispatch requires the utilization of transportation network data (discussed in Section II) and the integration of mobile resource modules into the existing optimization model. In this section, the distribution system module, DFTF module, and MESF module will be discussed in detail.

The flowchart of the integrated distribution system restoration is shown in Fig. 2. The grey-shaded processes are identical to traditional restoration flows without mobile resource dispatch. The only differences lie in the introduction of traffic data and the updated restoration model, marked as white-shaded processes in Fig. 2. Hence, the proposed mobile resource dispatch can be integrated into existing algorithms and implemented in real-world practices without much difficulty.

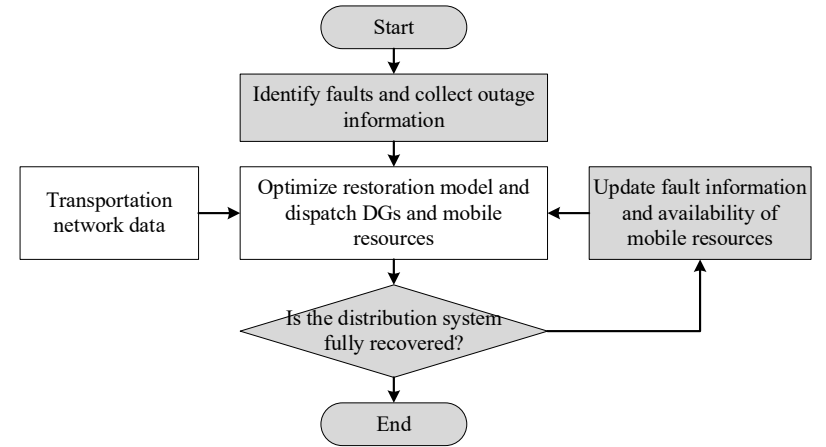

Fig. 2. Flowchart of integrated distribution system restoration process with mobile resource dispatch.

\section{B. Distribution System Module}

The convexified Distflow equations are employed to model the distribution system restoration model as shown in (12)-(24) [14]. The three-phase unbalance is ignored for simplicity, and the power loss and distribution line capacity are represented by quadratic constraints.

$$
\begin{gathered}
P_{i, t}^{\mathrm{G}}-P_{i, t}^{\mathrm{D}}=\sum_{i j \in \Phi_{\mathrm{L}}}\left(P_{i j, t}-P_{j i, t}+\Delta P_{i j, t} / 2\right) \\
Q_{i, t}^{\mathrm{G}}-Q_{i, t}^{\mathrm{D}}=\sum_{i j \in \Phi_{\mathrm{L}}}\left(Q_{i j, t}-Q_{j i, t}+\Delta Q_{i j, t} / 2\right) \\
\Delta P_{i j, t} \geq r_{i j}\left[\left(P_{i j, t}\right)^{2}+\left(Q_{i j, t}\right)^{2}\right] \\
\Delta Q_{i j, t} \geq x_{i j}\left[\left(P_{i j, t}\right)^{2}+\left(Q_{i j, t}\right)^{2}\right] \\
\left(V_{i, t}\right)^{2}-\left(V_{j, t}\right)^{2}=2\left(r_{i j} P_{i j, t}+x_{i j} Q_{i j, t}\right) \\
\left(V_{i}^{\mathrm{min}}\right)^{2} \leq\left(V_{i, t}\right)^{2} \leq\left(V_{i}^{\mathrm{max}}\right)^{2} \\
\left(P_{i j, t}\right)^{2}+\left(Q_{i j, t}\right)^{2} \leq \lambda_{i j, t}\left(S_{i j}\right)^{2} \\
P_{i, t}^{\mathrm{G}}=P_{i, t}^{\mathrm{RG}}+P_{i, t}^{\mathrm{DG}}+P_{i, t}^{\mathrm{ME}}+P_{i, t}^{\mathrm{D} \text { shed }} \\
Q_{i, t}^{\mathrm{G}}=Q_{i, t}^{\mathrm{RG}}+Q_{i, t}^{\mathrm{DG}}+Q_{i, t}^{\mathrm{ME}}+Q_{i, t}^{\mathrm{D}, \text { shed }} \\
0 \leq P_{i, t}^{\mathrm{D}, \text { shed }} \leq P_{i, t}^{\mathrm{D}} \\
Q_{i, t}^{\mathrm{D}, \text { shed }}=\varphi_{i}^{\mathrm{D}} Q_{i, t}^{\mathrm{D}} \\
0 \leq P_{i, t}^{\mathrm{RG}} \leq P_{i, t}^{\mathrm{RG}, \text { est }} \\
-\varphi_{i}^{\mathrm{RG}} P_{i, t}^{\mathrm{RG}} \leq Q_{i, t}^{\mathrm{RG}} \leq \varphi_{i}^{\mathrm{RG}} P_{i, t}^{\mathrm{RG}}
\end{gathered}
$$

where $P_{i, t}^{\mathrm{G}}\left(Q_{i, t}^{\mathrm{G}}\right), P_{i, t}^{\mathrm{D}}\left(Q_{i, t}^{\mathrm{D}}\right), P_{i j, t}\left(Q_{i j, t}\right)$, and $\Delta P_{i j, t}\left(\Delta Q_{i j, t}\right)$ denote the active (reactive) power generation, power load, power flow, and power loss, respectively. $r_{i j}$ and $x_{i j}$ denote the series resistance and reactance of distribution line, respectively. $V_{i, t}$, $V_{i}^{\max }$, and $V_{i}^{\max }$ denote the nodal voltage magnitude and its upper and lower limit, respectively. $\lambda_{i j, t}$ denotes the availability of line $i j$ at time $t$, and $S_{i j}$ denotes the apparent capacity of line $i j$, respectively. $P_{i, t}^{\mathrm{RG}}\left(Q_{i, t}^{\mathrm{RG}}\right), P_{i, t}^{\mathrm{DG}}\left(Q_{i, t}^{\mathrm{DG}}\right), P_{i, t}^{\mathrm{ME}}\left(Q_{i, t}^{\mathrm{ME}}\right)$, and $P_{i, t}^{\mathrm{D} \text {,shed }}\left(Q_{i, t}^{\mathrm{D} \text {,shed }}\right)$ denote the active (reactive) power generation of renewable DG, diesel generator, mobile energy storage, and load shedding, respectively. $P_{i, t}^{\mathrm{RG} \text {,est }}$ represents the forecasted renewable DG generation profile. $\varphi_{i}^{\mathrm{D}}$ and $\varphi_{i}^{\mathrm{RG}}$ denote the power factor of load and the power factor limit of the renewable DG converter, respectively.

Equations (12) and (13) denote the nodal power balance equations. The power losses are approximated by (14) and (15). Voltage drop is approximated by (16) and voltage magnitude is constrained by (17). Distribution line flow is limited by (18), and only available (undamaged/closed) lines have non-zero power flows. $P_{i, t}^{\mathrm{G}}$ and $Q_{i, t}^{\mathrm{G}}$ are further decomposed by (19) and (20). The amount of load shedding is limited by (21) and (22). The constraints of renewable DGs are enforced by (23) and (24).

\section{Integration of DFTF Module}

In addition to the transportation model of mobile resources discussed in Section II, the following constraints are introduced to integrate DFTF into distribution system restoration model:

$$
\begin{aligned}
& 0 \leq P_{i, t}^{\mathrm{DG}} \leq P_{i}^{\mathrm{DG}, \max } \\
& 0 \leq Q_{i, t}^{\mathrm{DG}} \leq \varphi_{i}^{\mathrm{DG}} P_{i, t}^{\mathrm{DG}} \\
& E_{i, t}^{\text {fuel }}=E_{i, t-1}^{\text {fuel }}-\alpha_{i} P_{i, t}^{\mathrm{DG}} \Delta t+E_{i, t}^{\text {fuel,in }}-E_{i, t}^{\text {fuel,out }} \\
& 0 \leq E_{i, t}^{\text {fuel }} \leq E_{i, t}^{\text {fuel,max }} \\
& E_{i, t}^{\mathrm{fuel}, \mathrm{in}}=\sum_{v \in \Phi_{\mathrm{V}}} \sum_{j \in \Phi_{\mathrm{N}, \mathrm{C}}} E_{v, j i, t}^{\mathrm{fuel}, \mathrm{in}} \\
& E_{i, t}^{\text {fuel,out }}=\sum_{v \in \Phi_{\mathrm{V}}} \sum_{j \in \Phi_{\mathrm{N}, \mathrm{C}}} E_{v, i j, t}^{\text {fuel,out }} \\
& E_{v, j i, t}^{\text {fuel,in }}=E_{v, j i, t-\Delta \tau_{i j}}^{\text {fuel out }} \\
& E_{v, j i, t}^{\text {fuel,out }} \leq y_{v, j i, t}^{\mathrm{D}} E_{v}^{\text {fuel,cap }}
\end{aligned}
$$

where $P_{i}^{\mathrm{DG} \text {,max }}$ denotes the maximum power output, and $\varphi_{i}^{\mathrm{DG}}$ denotes the power factor limit. $E_{i, t}^{\text {fuel }}$ denotes the diesel fuel reserve and is constrained by the maximum reserve denoted as $E_{i, t}^{\text {fuel,max }}$. The diesel fuel consumption is assumed to be a linear function of diesel generator output with $\alpha_{i}$ denoting the fuel consumption coefficient. $E_{i, t}^{\text {fuel,in }}$ and $E_{i, t}^{\text {fuel,out }}$ represent diesel fuel import and export, respectively. $E_{v, i j, t}^{\text {fuel,in }}$ and $E_{v, i j, t}^{\text {fuel,out }}$ denote the diesel fuel import and export from node $i$ to node $j$, respectively. $\Phi_{\mathrm{V}}$ denotes the set of DFTFs. The maximum capacity of the $v$-th DFTF is denoted as $E_{v}^{\text {fuel,cap }}$.

The active and reactive power outputs of diesel generators are constrained by (25) and (26). The diesel fuel reserve is calculated by (27) and constrained by (28). Diesel fuel transportation is constrained by (29)-(31) with respect to the transportation network. The capacity of a DFTF is limited by (32). 


\section{Integration of MESF Module}

The following constraints are introduced to integrate MESF into distribution system restoration model:

$$
\begin{gathered}
y_{w, i, t} P_{w}^{\mathrm{ME}, \mathrm{min}} \leq P_{w, i, t}^{\mathrm{ME}} \leq y_{w, i, t} P_{w}^{\mathrm{ME}, \max } \\
\left|Q_{w, i, t}^{\mathrm{ME}}\right| \leq \varphi_{w}^{\mathrm{ME}}\left|P_{w, i, t}^{\mathrm{ME}}\right| \\
P_{i, t}^{\mathrm{ME}}=\sum_{w \in \Phi_{\mathrm{ME}}} P_{w, i, t}^{\mathrm{ME}} \\
Q_{\mathrm{ME}}^{\mathrm{ME}}=\sum_{w \in \Phi_{\mathrm{ME}}} Q_{w, i, t}^{\mathrm{ME}} \\
E_{w, i, t}^{\mathrm{ME}}=E_{w, i, t-1}^{\mathrm{ME}}-\beta_{w} P_{w, i, t}^{\mathrm{ME}} \Delta t \\
y_{w, i, t} E_{w}^{\min } \leq E_{w, i, t}^{\mathrm{ME}} \leq y_{w, i, t} E_{w}^{\max }
\end{gathered}
$$

where $P_{w, i, t}^{\mathrm{ME}}\left(Q_{w, i, t}^{\mathrm{ME}}\right)$ denotes the active (reactive) power generation of the $w$-th MESF. Unlike diesel generators and renewable DGs, $P_{w, i, t}^{\mathrm{ME}}$ can be negative, meaning that MESF can consume power to charge the batteries. $P_{w}^{\mathrm{ME}, \max }$ and $P_{w}^{\mathrm{ME} \text {,min }}$ are the maximum power generation and consumption, respectively. $Q_{w, i, t}^{\mathrm{ME}}$ denotes the reactive power generation, and $\varphi_{w}^{\mathrm{ME}}$ denotes the power factor limit. $E_{w, i, t}^{\mathrm{ME}}$ and $\beta_{w}$ represent the energy storage level and battery efficiency, respectively. $E_{w}^{\max }$ and $E_{w}^{\min }$ denote the maximum and minimum energy storage level.

The active and reactive power outputs of MESFs are constrained by (33) and (34). The nodal power outputs from MESFs are calculated by (35) and (36). The energy storage level is calculated by (37) and constrained by (38).

\section{E. Integrated Model}

The objective function of the integrated distribution system restoration model is described in (39). The objective consists of three parts: load shedding penalty, total diesel fuel reserve level at the end of the time horizon (denoted as $T$ ), and the total energy storage level at $T$, respectively.

$$
\min \begin{gathered}
\sum_{t \in \Phi_{\mathrm{T}}} \sum_{i \in \Phi_{\mathrm{N}}} \theta_{i} P_{i, t}^{\mathrm{D}, \text { shed }}-\xi_{\mathrm{DG}} \sum_{i \in \Phi_{\mathrm{N}}} E_{i, T}^{\text {fuel }} \\
-\xi_{\mathrm{ME}} \sum_{i \in \Phi_{\mathrm{N}}} \sum_{w \in \Phi_{\mathrm{ME}}} E_{w, i, T}^{\mathrm{ME}}
\end{gathered}
$$

where $\theta_{i}$ denotes the penalty coefficient (weighting factor) of load shedding at different load nodes. $\xi_{\mathrm{DG}}$ and $\xi_{\mathrm{ME}}$ are weighting factors that are much smaller compared to $\theta_{i}$ to achieve minimal diesel fuel consumption and MESF discharge on condition that the load shedding can be minimized.

In summary, with mobile resources taken into account, the final model is formulated as a mixed-integer quadratically constrained program and can be described as:

Objective: (39)

Constraints: (3)-(38)

\section{F. MPC-Based Restoration for Uncertainty Modeling}

The travelling time matrix $\mathbf{T}, P_{i, t}^{\mathrm{RG}, \text { est }}$ of renewable DGs, and the availability of distribution lines $\left(\lambda_{i j, t}\right)$ are important input of the integrated restoration model. Yet, these parameters are significantly influenced by uncertainty factors such as traffic conditions, fault propagation, and weather forecasts. In this paper, MPC is employed to address the uncertain parameters. The developed MPC-based distribution system restoration is described by the following algorithm.

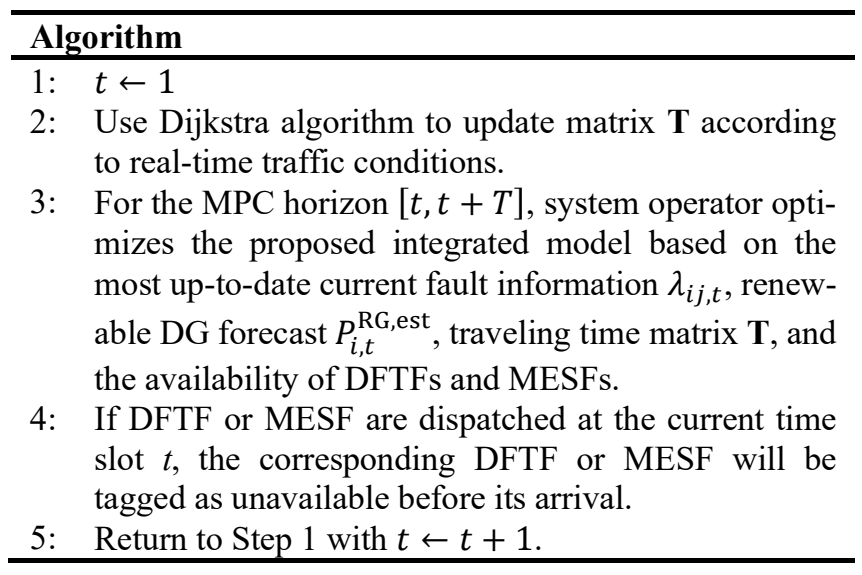

\section{CASe Study}

The IEEE 13-bus test feeder shown in Fig. 3 is employed to verify the proposed model [13]. The test feeder has two diesel generators located at nodes 5 and 8 . One solar DG and one wind DG are located at nodes 4 and 10, respectively. Each diesel generator has one DFTF vehicle, and two MESF vehicles are located at substation bus 1 when the fault occurs. The power loads at node 6 and node 11 are defined as important loads with the highest load shedding penalty. The MPC horizon is set to 3 hours with a time interval of 15 minutes. The simulation will also be conducted for 3 consecutive hours. Distribution line 910 will be tripped at the $5^{\text {th }}$ MPC interval due to unexpected fault, and line 7-9 will be repaired at the $8^{\text {th }}$ MPC interval, respectively. The proposed model is solved by CPLEX on a laptop computer with a quad-core i7 processor and 16-GB RAM.

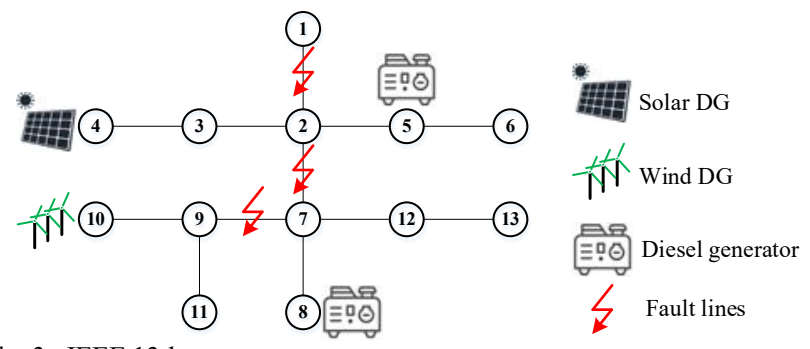

Fig. 3. IEEE 13-bus test system.

\section{A. Effectiveness Demonstration}

To evaluate the effectiveness of the proposed integrated distribution system model, the following two scenarios are simulated in this section: S-(i) power outage occurs at $9 \mathrm{AM}$ in the morning, and S-(ii) power outage occurs at $4 \mathrm{PM}$ in the afternoon. The major difference between these two scenarios is the trajectories of solar and wind generation. The statistics of these two scenarios are listed in Table I and the dispatch of mobile resources are summarized in Table II. 
TABle I StaTistics OF SimUlation RESUltS

\begin{tabular}{ccc}
\hline Scenario & S-(i) & S-(ii) \\
\hline Total MESF generation [MWh] & 1.067 & 0.530 \\
Total diesel generation [MWh] & 1.914 & 2.282 \\
Final MESF reserve [MWh] & 0.0 & 0.536 \\
Final diesel fuel reserve* [MWh] & 0.712 & 0.345 \\
Wind generation [MWh] & 1.438 & 1.846 \\
Solar generation [MWh] & 1.024 & 0.532 \\
Total load shedding [kWh] & 0.0 & 48.15 \\
Important load shedding [kWh] & 0.0 & 0.0 \\
CPU time [s] & 1.53 & 0.47 \\
\hline diesel fuel reserve is converted to MWh using diesel generator efficiency.
\end{tabular}

TABLE II DisPatCH OF MOBILE RESOURCES

\begin{tabular}{c|cc|cc||cc|cc}
\hline \multirow{2}{*}{$\begin{array}{c}\text { Time } \\
\text { slot }\end{array}$} & \multicolumn{2}{|c|}{ DFTF $v_{1}$} & \multicolumn{2}{c||}{ DFTF $v_{2}$} & \multicolumn{2}{c|}{ MESF $w_{1}$} & \multicolumn{2}{c}{ MESF $w_{2}$} \\
\cline { 2 - 8 } & S-(i) & S-(ii) & S-(i) & S-(ii) & S-(i) & S-(ii) & S-(i) & S-(ii) \\
\hline 1 & 8 & 8 & 5 & $5 \rightarrow 8$ & $1 \rightarrow 9$ & $1 \rightarrow 9$ & $1 \rightarrow 3$ & $1 \rightarrow 3$ \\
2 & $8 \rightarrow 5$ & $8 \rightarrow 5$ & 5 & 8 & $1 \rightarrow 9$ & $1 \rightarrow 9$ & 3 & 3 \\
3 & 5 & 5 & 5 & $8 \rightarrow 5$ & 9 & 9 & 3 & 3 \\
4 & $5 \rightarrow 8$ & $5 \rightarrow 8$ & $5 \rightarrow 8$ & 5 & 9 & 9 & $3 \rightarrow 11$ & 3 \\
5 & 8 & 8 & 8 & $5 \rightarrow 8$ & 9 & 9 & 11 & $3 \rightarrow 11$ \\
6 & 8 & 8 & $8 \rightarrow 5$ & 8 & 9 & 9 & 11 & 11 \\
7 & 8 & 8 & 5 & $8 \rightarrow 5$ & 9 & 9 & 11 & $11 \rightarrow 10$ \\
8 & 8 & 8 & 5 & 5 & 9 & 9 & 11 & 10 \\
9 & 8 & 8 & $5 \rightarrow 8$ & $5 \rightarrow 8$ & 9 & 9 & 11 & 10 \\
10 & 8 & 8 & 8 & 8 & 9 & 9 & 11 & 10 \\
11 & 8 & 8 & 8 & 8 & 9 & 9 & 11 & 10 \\
12 & 8 & 8 & 8 & 8 & 9 & 9 & 11 & 10 \\
\hline
\end{tabular}

Note that in Table II, ' $i$ ' and ' $i \rightarrow j$ ' represent that the fleet remains at node $i$ and the fleet is traveling from node $i$ to node $j$ at that time slot, respectively. For example, the statuses of DFTF $v_{1}$ in the first two time slots in scenario S-(i) are ' 8 ' and ' $8 \rightarrow 5$ ', indicating that $v_{1}$ remains parked at node 8 at time slot 1 and travels from node 8 to node 5 at time slot 2 , respectively.

As seen from Tables I and II, the DFTF $v_{1}$ and MESF $w_{1}$ have the same schedules in both scenarios, while the generation profiles of fixed DGs and the dispatch of $v_{2}$ and $w_{2}$ are considerably different. In scenario S-(i), load shedding can be completely alleviated and the diesel fuel reserve at the end of the MPC horizon is higher than that of scenario S-(ii). However, the energy stored in MESFs is completely exhausted. In scenario S-(ii), the solar generation capacity is significantly reduced compared to scenario S-(i), resulting in higher diesel fuel consumption. On the other hand, the wind power generation is redundant and one MESF is allocated to wind DG node 10 during MPC intervals 8-12 to absorb the redundant wind power generation according to Table II. Thus, MESFs still have energy reserve at the end of the horizon. Note that although diesel fuel and MESF storage are not completely used in scenario S(ii), load shedding is still inevitable due to the fluctuating generation capacity and failures of distribution lines.

As demonstrated in Table II, DFTFs are transporting diesel fuel from node 5 to node 8 in both scenarios because the diesel generator at node 8 has far less fuel reserve when the outage occurs. The fuel reserve level and the fuel import information at node 8 in these two scenarios are illustrated in Fig. 4.

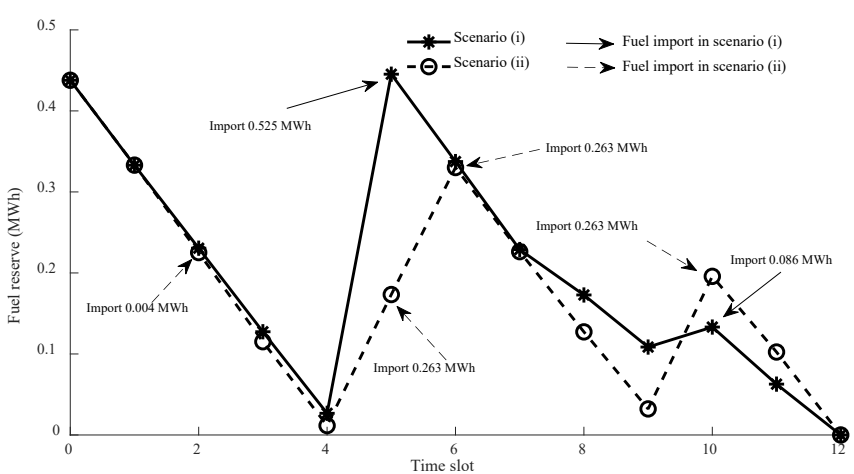

Fig. 4. Diesel fuel reserve profiles at node 8 in both scenarios.

\section{B. Comparisons}

To further verify the effectiveness of the proposed integrated model, the following comparing cases are simulated assuming the distribution system outage occurs at 4 PM:

Case 1: MPC with no mobile resources dispatch

Case 2: MPC with DFTF dispatch only

Case 3: MPC with MESF dispatch only

Case 4: One-run optimization with DFTF \& MESF dispatch

Case 5: MPC with DFTF \& MESF dispatch

The statistics of these 5 cases are compared in Table III. As shown in Table III, distribution system outage will cause a large amount of load shedding in case 1 if mobile resources are not considered. In case 2 , the total diesel generation is significantly boosted thanks to the introduction of DFTFs. Although the load shedding is improved in case 2 , the shedding of important loads is the same as in case 1 . The reason is that important load node 11 has no access to power sources from MPC intervals 4 to 8 when MESFs are not dispatched. Accordingly, the important load shedding can be successfully alleviated in case 3 by scheduling MESFs, but the load shedding is higher than that of case 2 due to the insufficient diesel fuel at node 8 . The difference between case 4 and case 5 is the method to handle uncertainties. Without MPC, case 4 cannot effectively respond to unexpected fault situations and renewable DG generation fluctuations, leading to a higher load shedding result. Case 5 represents the proposed method that achieves the lowest load shedding result. Hence, the proposed mobile resource dispatch model is validated to be efficient in assisting distribution system restoration.

TABLE III STATISTICS OF COMPARISON CASES

\begin{tabular}{ccccc}
\hline Case & $\begin{array}{c}\text { Total load } \\
\text { shedding } \\
{[\mathrm{kWh}]}\end{array}$ & $\begin{array}{c}\text { Important load } \\
\text { shedding } \\
{[\mathrm{kWh}]}\end{array}$ & $\begin{array}{c}\text { Total diesel } \\
\text { generation } \\
{[\mathrm{MWh}]}\end{array}$ & $\begin{array}{c}\text { Total MESF } \\
\text { generation } \\
{[\mathrm{MWh}]}\end{array}$ \\
\hline 1 & 1701.90 & 100.19 & 1.655 & 0.0 \\
2 & 368.66 & 100.19 & 2.627 & 0.0 \\
3 & 501.64 & 0.0 & 1.752 & 1.067 \\
4 & 114.19 & 4.47 & 1.910 & 0.826 \\
5 & 48.15 & 0.0 & 2.282 & 0.530 \\
\hline
\end{tabular}

V. CONCLUSIONS

This paper investigates the functions of mobile resources in assisting distribution system restoration after outages. The developed mobile resource dispatch model can be conveniently integrated into existing distribution system restoration models. The performance of the proposed integrated distribution system restoration model is validated through case studies. The introduction of mobile resource dispatch is demonstrated 
to be effective in reducing distribution system outage damages by minimizing the amount of load shedding.

\section{REFERENCES}

[1] F. Qiu and P. Li, "An integrated approach for power system restoration planning," Proc. IEEE, vol. 105, no. 7, pp. 1234-1252, Jul. 2017.

[2] C. P. Nguyen and A. J. Flueck, "Agent based restoration with distributed energy storage support in smart grids," IEEE Trans. Smart Grid, vol. 3, no. 2, pp. 1029-1038, Jun. 2012.

[3] V. Kumar, R. H. C. Kumar, I. Gupta, and H. O. Gupta, "DG integrated approach for service restoration under cold load pickup," IEEE Trans. Power Del., vol. 25, no. 1, pp. 398-406, Jan. 2010.

[4] J. Li, X. Y. Ma, C.-C. Liu, and K. P. Schneider, "Distribution system restoration with microgrids using spanning tree search," IEEE Trans. Power Syst., vol. 29, no. 6, pp. 3021-3029, Nov. 2014.

[5] Y. Wang, Y. Xu, J. He, et al., "Coordinating multiple sources for service restoration to enhance resilience of distribution systems," IEEE Trans. Smart Grid, vol. 10, no. 5, pp. 5781-5793, Sep. 2019.

[6] Y. Xu, C.-C. Liu, Z. Wang, K. Mo, K. P. Schneider, F. K. Tuffner, and D. T. Ton, "DGs for service restoration to critical loads in a secondary network," IEEE Trans. Smart Grid, vol. 10, no. 1, pp. 435-447, Jan. 2019.

[7] K. S. A. Sedzro, A. J. Lamadrid, and L. F. Zuluaga, "Allocation of resources using a microgrid formulation approach for resilient electric grids," IEEE Trans. Power Syst., vol. 33, no. 3, pp. 2633-2643, May 2018.

[8] L. Che and M. Shahidehpour, "Adaptive formulation of microgrids with mobile emergency resources for critical service restoration in extreme conditions," IEEE Trans. Power Syst., vol. 34, no. 1, pp. 742-753, Jan. 2019.

[9] S. Lei, J. Wang, C. Chen, and Y. Hou, "Mobile emergency generator pre-positioning and real-time allocation for resilient response to natural disasters," IEEE Trans. Smart Grid, vol. 9, no. 3, pp. 2030-2041, May 2018.

[10] W. Liu, Z. Lin, F. Wen, et al., "Sectionalizing strategies for minimizing outage durations of critical loads in parallel power system restoration with bi-level programming," Int. J. Elect. Power Energy Syst., vol. 71, pp. 327-334, 2015.

[11] S. Yao, P. Wang, and T. Zhao, "Transportable energy storage for more resilient distribution systems with multiple microgrids," IEEE Trans. Smart Grid, vol. 10, no. 3, pp. 3331-3341, May 2019.

[12] Q. Yan, B. Zhang, and M. Kezunovic, "The demand response support under weather impacts using PV generation and EV energy storage," in IEEE $16^{\text {th }}$ International Conference on Environment and Electrical Engineering (EEEIC), Florence, Italy, Jun. 2016, 6p.

[13] M. Carrion and J. M. Arroyo, "A computationally efficient mixed-integer linear formulation for the thermal unit commitment problem," IEEE Trans. Power Syst., vol. 21, no. 3, pp. 1371-1378, Aug. 2006.

[14] Q. Peng, Y. Tang, and S. H. Low, "Feeder reconfiguration in distribution networks based on convex relaxation of OPF," IEEE Trans. Power Syst., vol. 30, no. 4, pp. 1793-1804, Jul. 2015.

[15] Y. Lin, B. Chen, J. Wang, and Z. Bie, "A combined repair crew dispatch problem for resilient electric and natural gas system considering reconfiguration and DG islanding," IEEE Trans. Power Syst., vol. 34, no. 4, pp. 2755-2767, Jul. 2019. 